\title{
Wearable Systems for Industrial Augmented Reality Applications
}

\section{Christian Buergy \\ DHBW Mannheim}

Cooperative State University

Coblitzallee 1-9

68163 Mannheim GERMANY

christian.buergy@dhbw-mannheim.de

\section{Holger Kenn}

Microsoft

Advanced Technology Labs Europe

Rablstrasse 26

München GERMANY

Holger.Kenn@microsoft.com

\author{
Author Keywords \\ Wearable Computing; \\ Augmented Reality; \\ Industrial Applications; \\ Professional \\ Applications
}

\section{ACM Classification}

Keywords

H.5.1 Multimedia

Information Systems

\author{
Abstract \\ Augmented Reality (AR) is a successful application area \\ of Wearable Computing, especially for professional, \\ industrial settings, in which mobility is an important \\ factor. With the proliferation of mobile technology in the \\ workplace, wearable computing research can offer a \\ valuable contribution to the usability of mobile solutions, \\ such as the use of context information to inform devices \\ and services of the current task and user situation, \\ relieve professionals of tedious and repetitive \\ information entry tasks and increase worker safety in \\ complex and hazardous environments. Wearable AR \\ systems in general are widely utilized in various \\ domains, including architecture, military, tourism, \\ navigation, and entertainment. Such diverse usages \\ impose several challenges on researchers from both \\ areas of Augmented Reality and Wearable Computing, \\ such as interaction, activity and context recognition, \\ wearability, design, and modeling. For the second \\ Workshop on Wearable Systems for Industrial \\ Augmented Reality Applications, we have chosen the \\ motto "How to industrialize wearable AR?" We have \\ invited researchers from the relevant disciplines to \\ present novel works and discuss the applications of \\ state-of-the-art Wearable Computing research to \\ Augmented Reality systems. The workshop will provide \\ an opportunity for directed discussion sessions to \\ identify current issues, research topics, and solution
}


approaches, which lead to the proposal of future research directions.

\section{Objectives}

The objective of this workshop is to bring together researchers from academia, professional hardware and software developers and current and future users of wearable systems. We want to stimulate the application of Augmented Reality on wearable systems in professional environments.

In a previous first workshop, we set an agenda with research topics, which the participants defined as relevant for the topic. In this second workshop, we want to intensify this discussion and discuss in more detail the open research questions and the results and developments, which have been achieved since last year. We will review this preliminary list of relevant topics and have the goal to define a more detailed research roadmap or agenda for Wearable AR systems for the coming years. Another objective is to set up and keep an updated comparison between consumer application in the fields of Wearable Computing and Augmented Reality in contrast to industrial applications. The focus on industrial applications in this workshop shall not exclude the introduction of consumer-oriented systems, but rather seek transfer of knowledge and technology to be adapted into industrial applications as well as "consumerization" of originally industrial systems.

\section{Contributions}

At the workshop, Tim Verbellen and Bart Dhoedt from Ghent University and Pieter Simoens from Ghent University College will present their lightweight runtime environment for AR application that is described in their paper titled "Towards a Component-Based Platform for Industrial AR".

Matthias Berning, Till Riedel and Michael Beigl from the Karlsruhe Institute of Technology with Takuro

Yonezawa, Jin Nakazawa and Hide Tokuda from Keio University will present a system that allows to create prototypical 3D environments for rapid prototyping of AR applications. Their paper is titled "pARnorama: 360 Degree Interactive Video for Augmented Reality Prototyping".

Markus Ehrmann from the Munich University of Applied Sciences will present his research on user acceptance. His paper is titled "Evaluating Customer Expectance of Mixed Reality Applications in Order Picking".

Bernard Kress from Google and Meimei Shin from Nanoptron will present their work on optics for HMDs. Their paper is titled "Diffractive and holographic optics as optical combiners in Head Mounted Displays".

Christian Buergy from teXXmo and Mannheim Cooperative State University and Joerg Seitz from teXXmo will present lessons-learned of "The Bumpy Road of Bringing Wearable Augmented Reality Systems to Market".

Beyond these presented papers, we will have two or three invited talks of manufacturers of wearable AR systems, including a hands-on demo of their systems. In general, we encourage all presenters and participants to see this event as a hands-on workshop, in which system shall be demonstrated, tested and evaluated. This will definitely enrich the theoretical discussion of the R\&D requirements. 


\section{Outlook}

As in last year's workshop [1], we will encourage the discussion of open questions: The ambiguous motto of this year's workshop "How to industrialize Wearable $A R$ ?" shall both lead to a discussion of the challenges in applying todays wearable computing in industrial and professional settings as well as to a discussion the hurdles that need to be overcome to create tomorrows wearable AR industry with its own best practices, business models and value chains. The outcome of the discussions will be compiled in a consistent form and will be made available to the participants and on the workshop website [2].

\section{References}

[1] [Online]. Available: http://www.iswc.net/iswc12/ISWC2012 AdjunctProceedings.pdf [pp.75-135]

[2] [Online]. Available: http://www.cubeos.org/wearia13/. 